\author{
Publications from the Humboldt Kolleg Ecuador 2019 \\ "Breaking Paradigms: Towards a Multi-, Inter- and \\ Transdisciplinary Science" \\ In commemoration of the 250th Anniversary of \\ February 21th - 24th, 2019. \\ 250 \\ Ibarra, Ecuador

\section{Alexander von Humboldt \\ Stiftung / Foundation}

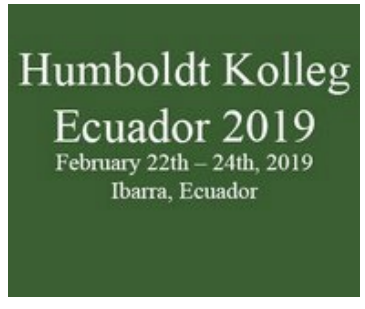

Humboldt Kolleg

Ecuador 2019

Ibarra, Ecuador

\section{Bionatura Conference Series Vol 2. No 1. 2019}

"Breaking Paradigms: Towards a Multi-, Inter- and Transdisciplinary Science" In commemoration of the 250th Anniversary of Alexander von Humboldt

REVISION/REVIEW

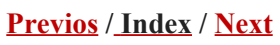

\title{
Anti-angiogenesis therapy for the treatment ovarian cancer
}

\author{
Alejandra Cevallos and Abigail Solórzano \\ available in: http://dx.doi.org/10.21931/RB/CS/2019.02.01.26
}

\begin{abstract}
The process of angiogenesis is regulated by several growth factors, the most important factor is VEGF which is an important mediator because it facilitates the growth of blood vessels and the remodeling processes. In addition, angiogenesis is significantly involved in tumor progression giving the tumor the necessary supplies for its survival and thus causing metastases in other organs by providing a site of entry to the circulation of vessels blood. The objective of this review is to reflect the fundamental role that angiogenesis has in the development and spread of cancer through vascular endothelial growth factor, its receptors, and its signaling pathways. On the other hand, antiangiogenics strategies (angiogenesis inhibitors) are proposed to fight cancer and clinical trials are presented to verify the survival range of patients who have been treated with anti-angiogenic therapy.
\end{abstract}

Keywords: Angiogenesis, anti-angiogenesis, VEGF, inhibitors, cancer

\section{INTRODUCTION}

Angiogenesis is the formation of new blood vessels from other pre-existing ones. It is a process that is already inhibited when the human being reaches its adult stage, however, it can be activated in response to a physiological process or by a tissue damage, for example, in wound healing. ${ }^{1}$ It was demonstrated that there are different molecules involved in the positive regulation of angiogenesis, including the vascular endothelial growth factor. VEGF is a homodimeric glycoprotein with specific mitogenic action of endothelial cells, it is the main molecule in the intervention of neovascularization. So far, it have identified three high affinity receptors for this factor: VEGF-R1 or 
Fit-1, VEGF-R2 or FLK-1/KDR and VEGF-RB, which are receptors of tyrosine kinases. ${ }^{2}$ Therefore, VEGF is an important mediator in angiogenesis, as it facilitates the growth of blood vessels and remodeling processes. ${ }^{3}$ On the other hand, angiogenesis was identified as an important process in cancer growth. Angiogenesis does not participate in carcinogenesis, but if it promotes tumor progression, it provides the oxygen supply tumor and nutrients necessary for the tumor to grow ${ }^{4}$ and may cause metastases in other organs.

In the last years years, a challenge in medicine has been to find pharmaceutical agents that make tumor cells more sensitive to chemotherapy, avoiding the creation of toxic effects in the organism. ${ }^{5}$ For this, several clinical trials were carried out by putting to testing anti-angiogenic drugs, developed through the study of the molecules involved in antiangiogenesis. Then they different ways of inhibiting angiogenesis have been distinguished, it has been demonstrated that agents that attack the VEGF molecule can inhibit neovasculature.

We will know about two types of drugs or molecules that have helped to reduce or prevent metastasis in ovarian cancer. The first is a monoclonal antibody that is responsible for interrupting the binding between VEGF and its receptor and the second is a recently found tyrosine kinase inhibitor. It was evaluated its efficacy through the study of one clinical trials, administering and evaluating its progress after consuming the drug.

\section{ANGIOGENESIS}

According to Martínez, J y Herrera, L 2 : Angiogenesis is carried out mainly by the following steps: (1) The endothelial cells (ECs) precursors give origin to the blood vessels; (2) ECs are assembled forming a primitive vascular maze of small capillaries; (3) The vascular plexus expands progressively due to the birth of the vessels; (4) which is remodeling forming a highly organized vascular network of large vessels branching into small vessels; (5) The newly formed channels by ECs are covered by pericytes and smooth muscle cells, which regulate the contraction and dilatation of the blood vessels, providing resistance and allowing the regulation of the perfusion of the vessels. ${ }^{2}$

Angiogenesis can be physiological (normal) or pathological (abnormal); The physiological is produced in the healing of the wounds and in the reproductive cycle female, and the pathological one appears when it reaches the limit or exceeds the limit between the activating and inhibiting molecules, and takes place during the tumor growth, rheumatoid arthritis and diabetic retinopathy proliferative. ${ }^{2,6}$

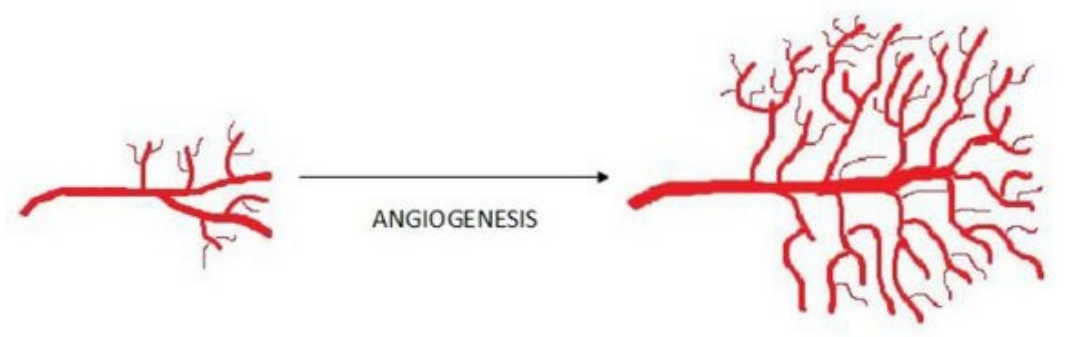

Figure 1. Process of angiogenesis

\section{ANGIOGENESIS IN CANCER}

Tumor angiogenesis is the proliferation of a group of new blood vessels that penetrate into the inside of the tumor, providing oxygen and nutrients. This occurs because the tumor and host cells unload chemicals near the tumor. The process of angiogenesis is regulated by activating and inhibiting molecules. ${ }^{7}$

In normal conditions predominates the inhibition. And when the need for a new vascularization is presented, the activators of the angiogenesis increase and the inhibitors decrease. 
This causes the secretion of molecules by part of the tumor that send signals to the surrounding healthy tissue. This signal activates certain genes of the host tissue, which causes the stimulation of proteins for the growth of new blood vessels. These new vessels are neither functional nor morphologically normal. These vessels are weak, blind and their flow is inverted and discontinuous. ${ }^{7}$

As a result, although there is an increase in vascularization, the intake of oxygen and drugs is scarcer than in healthy tissue. This contributes to the genetic selection of tumor cells and the resistance to drugs and radiation. ${ }^{7}$

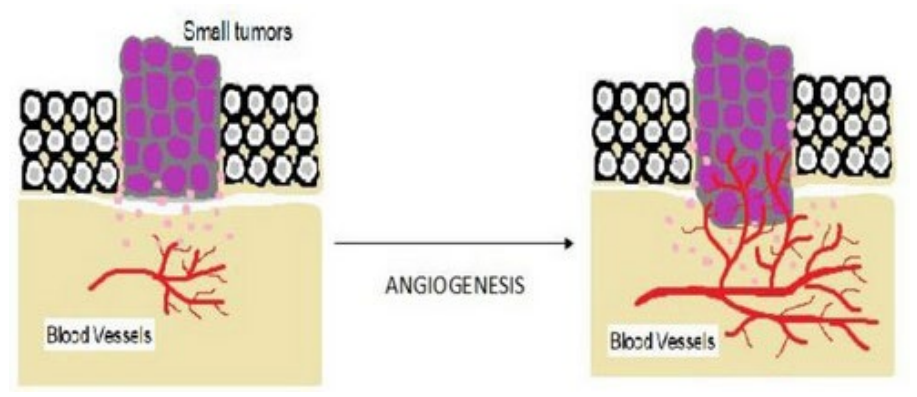

Figure 2. Process of angiogenesis in cancer.

Angiogenesis does not participate in carcinogenesis, but if it promotes tumor progression and is fundamental to the formation of metastases by providing a site of entry to the circulation of blood vessels, as well as oxygen and nutrients by angiogenesis. ${ }^{3}$

\section{GROWTH FACTORS ANGIOGENIC.}

There are 12 angiogenic proteins currently recognized. The most commonly identified in tumors are fibroblast growth factor (FGF) and vascular endothelial growth factor (VEGF). ${ }^{1}$

\section{Vascular Endothelial Growth Factor (VEGF).}

VEGF is a homodimeric glycoprotein of between 34 and $46 \mathrm{kDa}$ with specific mitogenic action of endothelial cells. ${ }^{6}$ VEGF is normally produced during embryogenesis and in adult life in normal and pathological processes, including tumor development. Currently, VEGF and its receptors are recognized as the main regulator of the ECs vascular and the formation of blood vessels. ${ }^{6,8}$

The VEGF family includes VEGF-A, VEGF-B, VEGF-C, VEGF-D, VEGF-E and placental growth factor (PlGF). All of them are located on different chromosomes, but they share a considerable homology. In addition, VEGF-A exists in four isoforms. VEGF121, VEGF165, VEGF189, and VEGF206 are generated by alternative mRNA splicing. 8

According to Binda, $\mathrm{M}^{6}$ "Most tumors overexpress the VEGF factor. In malignant tumors and in wounds usually occurs the hypoxia that increases the transcription of the gene that encodes for VEGF and makes the messenger RNA more stable once that occurred. During hypoxia, is produces a molecule called transcription factor 1 inducible by hypoxia (HIF-1) that is translocated to the nucleus and sticks to a nucleotide sequence called hypoxia response element (HRE) located in the region 5' promoter of gene VEGF. The binding of HIF-1 to HRE initiates the transcription of the gene. Solid tumors usually grow up to $1 \mathrm{~mm}$ in diameter, coming to them the nutrients by simple 
diffusion. Beyond that volume, simple diffusion is not sufficient and hypoxia is generated in the center of the tumor, thus increasing the expression of VEGF. The production of this factor produces the growth of new blood vessels towards the tumor."

Therefore, VEGF is an important mediator in angiogenesis, because it facilitates the growth of blood vessels and remodeling processes, as well as providing mitogenic and survival stimuli for endothelial cells. ${ }^{3}$

\section{VEGF receptors}

Members of the VEGF family are characterized by different interactions with three receptors of tyrosine kinase, that include the VEGFr-1 (FLT-1), VEGFr-2 (FLK-1/KDR) and VEGFr-3 and two non-protein receptors of tyrosine kinase that including Neuropilin 1 and Neuropilin 2. Hypoxia induces the formation of VEGF by the EC and produces an increase in the number of VEGF receptors. ${ }^{2}$ The main receivers are:

- VEGFr-1 generates signals that organize the assembly of the EC in functional tubes and vessels. ${ }^{8}$

- VEGFr-2 is responsible for the proliferation and migration of EC. ${ }^{8}$

- VEGFr-3 (FLT-4) has the function of maintaining the ECs coating during tumour angiogenesis. ${ }^{2}$

\section{ANTI-ANGIOGENIC THERAPY IN CANCER TREATMENTS}

The inhibition of angiogenesis has been investigated for a long time as an important goal to fight cancer. Achieving as a first step to identify all the molecules involved in the angiogenic process, however, it should be taken into account which are the most important and whose regulatory capacity on vascular proliferation both intervenes in the stimulation as the inhibition.

The anti-angiogenesis involves a therapy against stimulating molecules in the neovasculature, with the intention of inhibiting the formation and expansion of blood vessels, ${ }^{9}$ especially used in cancer therapies. Many antiangiogenics techniques are being developed based on the inhibition of endothelial cell function (e.g., inhibitors of the Metaloproteasas matrix, MMP) and others based on directly attacking the specific molecules involved in the formation of blood vessels ${ }^{10}$ as the Vascular Growth factor (VEGF). The VEGF inhibitory technique has become the main objective ${ }^{9}$ because this technique has shown positive effects against tumors in vivo tests on animals.

As we previously saw the Vascular endothelial growth factor (VEGF) is a key mediator of normal physiological angiogenesis, in the same way, acts as an important molecule of tumor angiogenesis, stimulates the growth of new blood vessels and in this way the tumor can be supplied with oxygen and nutrients needed to continue its growth. ${ }^{3}$ Therefore, blocking the action of VEGF seems to be one of the best ways to attack tumors, has been investigated several ways to mitigate the role of this growth factor and thus avoid angiogenesis, among the most investigated we have the monoclonal antibodies against VEGF receptors. Also, are inhibitors that bind to VEGF and/or its receptor on the surface of endothelial cells by blocking their activity ${ }^{11}$ and low molecular weight tyrosine kinase (TKI) inhibitors. Each of these strategies is being investigated in clinical trials.

\section{Monoclonal antibodies against VEGF}

One of the most studied clinical trials to inhibit the key factor of angiogenesis (VEGF) is through monoclonal antibodies. In 2004 was approved bevacizumab in the United States by the Food and Drug Administration (FDA). ${ }^{12}$ 
with its receptors Flt-1 (VEGFR-1) and KDR (VEGFR-2), ${ }^{12}$ inhibiting the activity of VEGF occurs a regression of the tumor and prevents a new formation of blood vessels to help the growth of the tumor. Bevacizumab or also called Avastin is applied for the treatment of colorectal and lung cancer initially, however, when discovering the presence of VEGF factor in ovarian and prostate cancer, studies and tests were started to apply the drug in the two types of cancer and as mentioned by the American Cancer Society 13 "Bevacizumab has shown that it shrinks or decreases the growth of advanced-stage epithelial ovarian cancers." On the other hand, it has been found that this drug has a better activity if it is administered along with chemotherapy. ${ }^{14}$ However, all drugs bring side effects, the most frequent and mild in patients treated with this medicine is hypertension and the most serious is the gastrointestinal perforation that can lead to the death of the patient. ${ }^{15}$

\section{Tyrosine Kinase Inhibitors (TKI)}

Another important way to inhibit the activity of the Vascular endothelial growth factor is through different molecules that can recognize and inhibit the receptors of tyrosine kinase. Its objective is to block the intracellular signaling induced by the activation of the tyrosine kinase receptor (VEGFR), ${ }^{2}$ inhibitors are responsible for blocking directly the changes that occur in the intracellular domain. The table 1 shows the most used drugs for cancer therapy, using as target molecules these involved in the process of angiogenesis, inhibiting their ability to create new blood vessels.

\begin{tabular}{|c|c|c|c|}
\hline \multicolumn{4}{|c|}{ Tyrosine kinase inhibitors } \\
\hline Inhibitors & Target Molecule & $\begin{array}{l}\text { Administration } \\
\text { routes }\end{array}$ & Types of Cancer \\
\hline Erlotinib & EGFR & Via Oral & Lung cancer and pancreatic cancer ${ }^{16}$ \\
\hline Gefitinib & EGFR & Via Oral & Non-microcytic lung cancer ${ }^{16}$ \\
\hline Osimertinib & EGFR & Via Oral & Non-small cell lung cancer (NSCLC) ${ }^{16}$ \\
\hline Imatinib & $\begin{array}{l}\text { bcr-abl protein, c-kit receptor, } \\
\text { PDGFR }\end{array}$ & Via Oral & $\begin{array}{l}\text { Chronic myeloid leukemia } \mathrm{Ph}+ \\
\text { Gastrointestinal stroma or } \mathrm{GIST}^{16}\end{array}$ \\
\hline Apatinib & VEGFR & Via Oral & $\begin{array}{l}\text { Gastric cancer, colorectal cancer non- } \\
\text { small cell lung cancer (NSCLC) }{ }^{16}\end{array}$ \\
\hline Sunitinib & PDGFR c-kit, VEGFR & Via Oral & $\begin{array}{l}\text { Cancer Gastrointestinal stroma or GIST, } \\
\text { kidney cancer }{ }^{2,15,17}\end{array}$ \\
\hline Sorafenib & PDGFR c-kit, Raf, VEGFR & Via Oral & Kidney cancer $^{2}$ \\
\hline Axitinib & VEGFR, PDGFR y c-Kit & Via Oral & Metastatic renal cancer ${ }^{16,17}$ \\
\hline Pazopanib & VEGFR, PDGFR y c-Kit & Via Oral & Advanced renal cell carcinoma ${ }^{16,17}$ \\
\hline Lenvatinib & $\begin{array}{l}\text { VEGFR-1-3, FGFR-1-4, RET, c- } \\
\text { KIT, PDGFR } \alpha\end{array}$ & Via Oral & Thyroid cancer ${ }^{16}$ \\
\hline Vandetanib & VEGFR y EGFR & Via Oral & $\begin{array}{l}\text { Symptomatic medullary thyroid } \\
\text { cancer }^{16,17}\end{array}$ \\
\hline Regorafenib & VEGFR1, $-2,-3$, KIT, RET, RAF-1 & Via Oral & Colorectal cancer ${ }^{16}$ \\
\hline
\end{tabular}

\section{Table 1: VEGF RTKIs and the cancer types where these drugs could act}

Angiogenesis plays a fundamental role in the growth of tumors, for this reason, the mitigation or inhibition of VEGF could be a solution to avoid a metastasis of cancer or even to make that all the cancer cells die.

\section{OVARIAN CANCER}

This type of cancer is originating by the ovaries, although some ovarian cancers can start in the fallopian tubes and it spread. Usually this type of cancer appears in late stages, when women arrive at the menopause stage. ${ }^{18}$

Ovaries are an important part of the female reproductive system. Its function is to secrete ovules and produce sex hormones. This type of cancer can be caused by genetic, hormonal or reproductive factors. ${ }^{19}$ For example, it can be 
caused by a hereditary genetic mutation of the genes BRCA1 and BRCA2, these mutations were identified in people who previously had breast cancer and it is estimated that more than $10 \%$ of cancers of this type are caused by this mutation. ${ }^{19}$ In addition, these mutations increase the risk of acquiring another type of cancer such as fallopian tube carcinoma. $^{19}$

Ovarian cancer in its early stages is difficult to detect because it does not have visible symptoms. And when these symptoms become notorious is because the cancer is already advanced and at that time it is difficult to treat it. The most common symptoms are the pains in the lower abdomen and the vaginal hemorrhages. ${ }^{20}$

According to Rivas, LM 18 "Ovarian cancer is the fifth leading cause of death in women. Approximately 50\% of women who are diagnosed with ovarian cancer are above the age of 62 years. White women are more likely to have ovarian cancer than African American women. The American Cancer Society estimates that in 2016, in the United States, there will be approximately 22.280 new cases of ovarian cancer and approximately 14.240 people will die from it."

\section{ANGIOGENESIS INHIBITORS IN CLINICAL TRIALS}

Several clinical essays have been realized to verify the ability of drugs to inhibit angiogenesis and thus prevent the spread of cancer. We analyze one study with a way of inhibiting VEGF, it was done by applying monoclonal antibodies as Bevacizumab.

In this study, we will discuss patients with ovarian cancer who were subjected to a treatment with bevacizumab. The study involved 1873 women from different states suffering from epithelial ovarian cancers, more than $80 \%$ had tumor Grade 3 serous adenocarcinomas, ${ }^{21}$ none were previously treated and neither underwent surgery for the eradication of cancer. It was a treatment of 22 cycles in 3 weeks where the patients were randomly divided into 3 groups, the first group was composed of 625 women and were the control group, the second group of similar form were 625 women and were the therapy of initiation with bevacizumab and the last group formed by 623 women obtained the full dose Bevacizumab in all their treatment.

The three groups were subjected to chemotherapy with carboplatin and paclitaxel $(175 \mathrm{mg} / \mathrm{m} 2)$ in the first 6 cycles, their only difference was the additional compound. In group 1, an additional placebo was applied since cycle 2 and then the placebo was applied throughout the treatment. In group 2 additional to chemotherapy was applied Bevacizumab in the first 6 cycles and then only apply placebo and in group 3 was applied chemotherapy along with Bevacizumab, but the drug from cycle 7 onwards only applied the Bevacizumab $(15 \mathrm{mg} / \mathrm{kg})$.

The results of this study were a significant improvement in progression-free survival. ${ }^{21}$ When the patients were subjected to therapy of bevacizumab along with the chemotherapy and then single dose of bevacizumab compared with therapy control only with placebo and with chemotherapy alone, this resulted in the $28 \%$ reduction in the risk of progression. ${ }^{21}$ In addition, there were differences in other aspects such as gastrointestinal perforation rates where in this study it was observed that the groups that received the drug suffered from gastrointestinal perforation rather more than the control group, however, the rate of concerned was still less than people with tumors not gynecological. Although about $66 \%$ of women in the study abandoned treatment before finishing for reasons such as progression of the disease or by side effects caused by the drug, it was possible to verify greater effectiveness of the drug Bevacizumab along with chemotherapy.

\section{CONCLUSION}

In order to understand antiangiogenesis, first had to understand how angiogenesis works or intervenes in the 
propagation of tumors. The role of the angiogénesis was examined in the process of development of cancer, knowing all the molecules and receptors in addition to the intracellular signaling involved during the neovasculature. The VEGF was taken as a fundamental molecule, by its capacity to intervene in both the stimulation as in the inhibition of the neovascularization. By knowing all the processes by which this molecule must pass to achieve the creation of blood vessels, it would provide several strategies to interfere in the process. Two different molecules inhibitors of angiogenesis were presented, the first was monoclonal antibodies capable of interfering with the process of binding between VEGF and its receptor in such a way that it cannot be started with angiogenesis. For example, Bevacizumab is an antibody elaborated for this purpose for this reason its efficacy was assessed by a clinical study. The second was tyrosine kinase inhibitor (TKI) molecules that act by intervening in the intracellular signaling and thus avoid the creation of new blood vessels. With the clinical trials analyzed, it was concluded that angiogenesis inhibitors in combination with chemotherapy may produce better therapeutic benefits in the treatment of ovarian cancer.

\section{REFERENCES}

1. Centro Nacional de Información de Ciencias Médicas. V. Revista cubana de investigaciones biomédicas. [Internet]. Vol. 20, Revista Cubana de Investigaciones Biomédicas. Centro Nacional de Información de Ciencias Médicas, Ministerio de Salud Pública; 1982 [cited 2019 May 14]. 223-230 p. Available from: http://scielo.sld.cu/scielo.php?script=sci_arttext\&pid=S0864-03002001000300010

2. Martínez-Ezquerro, J. D.; Herrera, L. A. Angiogénesis: VEGF/VEGFRs como blancos terapéuticos en el tratamiento contra el cáncer. Cancerología. 2006; 1(1), 83-96.Martínez-Ezquerro, J. D.; Herrera, L. A. Angiogénesis: VEGF/VEGFRs como blancos terapéuticos en el tratamiento contra el cáncer. Cancerología. 2006; 1(1), 83-96.

3. Carmeliet P. VEGF as a key mediator of angiogenesis in cancer. Oncology. 2005;69(SUPPL. 3):4-10.

4. Stoeltzing O, Liu W, Reinmuth N, Parikh A, Ahmad SA, Jung YD, et al. New approaches to the treatment of hepatic malignancies: Angiogenesis and antiangiogenic therapy of colon cancer liver metastasis. Ann Surg Oncol. 2003;10(7):722-33.

5. RS K. Antiangiogenic therapy: A universal chemosensitization strategy for cancer? Science (80- ). 2006;312(May):1171.

6. Binda MM. Factores reguladores de la angiogénesis en el fenómeno de resistencia antitumoral concomitante. 2002;134.

7. Sociedad Española de Oncología Médica. ANGIOGÉNESIS - SEOM: Sociedad Española de Oncología Médica (C) 2019 [Internet]. 2006 [cited 2019 May 13]. p. 4. Available from: https://seom.org/43-Socios - Formación y Recursos/Bases de la Oncología/157-angiogenesis

8. Vale PR, Losordo DW, Symes JF, Isner JM. Factores de crecimiento para la angiogénesis terapéutica en las enfermedades cardiovasculares. Rev Española Cardiol. 2013;54(10):1210-24.

9. Harris AL. Antiangiogenesis for cancer therapy. Lancet. 2004;349:S13-5.

10. Rosen L. Antiangiogenic Strategies and Agents in Clinical Trials. Oncologist. 2004;5(90001):20-7.

11. National Cancer Institute. Inhibidores de la angiogénesis [Internet]. [cited 2019 May 15]. Available from: https://www.cancer.gov/espanol/cancer/tratamiento/tipos/inmunoterapia/hoja-informativa-inhibidores-angiogenesis

12. Del M, Montes-Vera R, Garrido-Acosta O, Anguiano-Robledo L, Sánchez-Navarrete J, Pérez-Cruz E, et al. Aspectos farmacocinéticos de bevacizumab. Rev Hosp Jua Mex [Internet]. 2013;80(1):73-8. Available from: http://www.medigraphic.com/pdfs/juarez/ju-2013/ju1311.pdf 
13. Terapia dirigida para el cáncer de ovario [Internet]. [cited 2019 May 15]. Available from: https://www.cancer.org/es/cancer/cancer-de-ovario/tratamiento/terapia-dirigida.html

14. Pujade-Lauraine E, Hilpert F, Weber B, Reuss A, Poveda A, Kristensen G, et al. Bevacizumab combined with chemotherapy for platinum-resistant recurrent ovarian cancer: The AURELIA open-label randomized phase III trial. J Clin Oncol. 2014;32(13):1302-8.

15. Shahi PK, Rueda ADELC, Manga GP. Angiogénesis neoplásica. An Med Interna. 2008;25(7):366-9.

16. Sociedad Española de Oncología Médica, Nuevos tratamientos biológicos: qué son y cómo actúan SEOM: Sociedad Española de Oncología Médica (C) 2019 -[Internet]. [cited 2019 May 17]. Available from: https://seom.org/guia-actualizada-de-tratamientos/nuevos-tratamientos-biologicos-que-son-y-como-actuan?start=3

17. Shojaei F. Anti-angiogenesis therapy in cancer: Current challenges and future perspectives. Cancer Lett [Internet]. 2012;320(2):130-7. Available from: http://dx.doi.org/10.1016/j.canlet.2012.03.008

18. Rivas-Corchado LM, González-Geroniz M, Jorge Hernández-Herrera R. 558 GinecoloGía y obstetricia de México Perfil epidemiológico del cáncer de ovario Artículo original [Internet]. [cited 2019 May 15]. Available from: www.nietoeditores.com.mx

19. Zalduendo P. Cáncer de ovario: Síntomas, cómo se detecta y supervivencia [Internet]. Vidasana CUN. 2018 [cited 2019 May 23]. Available from: https://www.enfermedadesgraves.com/blog/cancer-de-ovario/

20. Cáncer de ovario. [cited 2019 May 23]; Available from: https://medlineplus.gov/spanish/ovariancancer.html

21. Fleming GF, Monk BJ, Huang H, Mannel RS, Homesley HD, Fowler J, et al. Incorporation of Bevacizumab in the Primary Treatment of Ovarian Cancer. 2011;2473-83.

Received: 17 April 2019

Accepted: 23 May 2019

Alejandra Cevallos and Abigail Solórzano

Genetic Engineering, School of Biological Sciences and Engineering,

YachayTech, Urcuquí. Ecuador.

alejandra.cevallos@yachaytech.edu.ec

abigail.solorzano@yachaytech.edu.ec 